\title{
Clinical Guideline Highlights for the Hospitalist: Management of Acute and Chronic Pain in Sickle Cell Disease
}

\author{
Charles D Pham, MD*, Duong T Hua, MD
}

Department of Medicine, Division of Hospital Medicine, Harbor-UCLA Medical Center, Torrance, California.

GUIDELINE TITLE: American Society of Hematology 2020 Guidelines for Sickle Cell Disease: Management of Acute and Chronic Pain

RELEASE DATE: June 19, 2020

PRIOR VERSION: Not applicable
DEVELOPER: American Society of Hematology Guideline Panel on Sickle Cell Disease-Related Pain

FUNDING SOURCE: American Society of Hematology

TARGET POPULATION: Adult and pediatric patients with a history of sickle cell disease with acute and chronic pain. ickle cell disease (SCD) affects an estimated 100,000 people in the United States. ${ }^{1}$ Pain is the most common complication of SCD and the primary reason patients with SCD seek medical attention. ${ }^{2}$ In 2016, three-fourths of the approximately 130,000 SCD-related hospitalizations in the United States involved pain crises. ${ }^{3}$ When managing patients with SCD and chronic pain, an individualized and interdisciplinary approach is crucial. In 2020, the American Society of Hematology (ASH) developed guidelines reflecting the latest evidence in managing acute and chronic pain in adult and pediatric patients with SCD. The ASH guidelines provide 18 recommendations; here, we highlight the 8 recommendations most pertinent to the hospitalist.

\section{KEY RECOMMENDATIONS FOR THE HOSPITALIST}

\section{Acute Pain}

Acute pain in the guideline is defined as pain that results in an unplanned visit to an acute care center for treatment.

Recommendation 1. For adult and pediatric patients presenting to an acute care setting with SCD-related acute pain, the $\mathrm{ASH}$ guideline panel recommends rapid (ie, within 1 hour of arrival at the emergency department [ED]) assessment and administration of analgesia, with reassessments every 30-60 minutes to optimize pain control (Strong recommendation; low certainty in the evidence about effects).

Although the perceived benefits are unclear due to insufficient evidence, the panel agrees that delaying pain management results in undeniable harm to patients. Hence, this recommendation was deemed both acceptable and ethical. Rapid evaluation also allows for earlier identification and treatment of other potential SCD-related complications.

*Corresponding Author: Charles D Pham, MD; Email: cpham@dhs.lacounty.gov; Telephone: 424-306-4000.

Published online first March 17, 2021.

Received: August 31, 2020; Revised: October 23, 2020; Accepted: October 26, 2020

() 2021 Society of Hospital Medicine DOI 10.12788/jhm.3556
Recommendation 2. For adult and pediatric patients presenting to an acute care setting with SCD-associated pain for whom opioid therapy is indicated, the ASH guideline panel suggests tailored opioid dosing based on consideration of baseline opioid therapy and prior effective therapy. (For adults: conditional recommendation; moderate certainty in the evidence about effects. For children: conditional recommendation; low certainty in the evidence about effects).

One randomized controlled trial examined patient-specific opioid dosing (based on current chronic opioid therapy [COT] and previously known effective acute pain management) vs weight-based dosing in the ED and found that participants randomized into the patient-specific protocol had a greater reduction in pain and decreased rate of hospital admission. ${ }^{4}$

The panel acknowledges that intravenous patient-controlled opioid analgesia is generally the standard of care at most institutions. However, no clear data address whether continuous opioid infusion in addition to on-demand dosing is beneficial.

Recommendation 3. For adult and pediatric patients with acute pain related to $S C D$, the ASH guideline panel suggests a short course (5 to 7 days) of nonsteroidal anti-inflammatory drugs (NSAIDs) in addition to opioids (Conditional recommendation; very low certainty in the evidence about effects).

The use of NSAIDs for managing pain in hospitalized patients with SCD has been associated with a reduction in the use of opioids in the inpatient setting and decreased lengths of stay. ${ }^{5}$ The potential harms of NSAIDs, including renal and gastrointestinal toxicity, however, should be factored into the decision-making as the risks may outweigh the potential benefits.

Recommendation 4. For adult and pediatric patients with SCD hospitalized for acute pain, the ASH guideline panel suggests a subanesthetic (analgesic) infusion of ketamine as adjunctive treatment of pain refractory or not effectively treated with opioids alone (Conditional recommendation; very low certainty in the evidence about effects). The guideline panel also suggests regional anesthesia for localized pain refractory or not effectively treated with opioids alone (Conditional recommendation; very low certainty in the evidence about effects).

Studies have demonstrated reduced pain and opioid utiliza- 
tion in individuals who received adjuvant ketamine infusions ${ }^{6}$ or regional anesthesia (ie, epidural). ${ }^{7}$ Feasibility, however, is limited to centers that have the appropriate experience and expertise with these interventions.

Recommendation 5. For adult and pediatric patients who have recurrent acute pain associated with SCD, the ASH guideline panel suggests against chronic monthly transfusion therapy as a first-line strategy to prevent or reduce recurrent acute pain episodes (Conditional recommendation; low certainty in the evidence about effects). The evidence for monthly transfusions in preventing recurrent pain is limited. There is, however, a moderate risk of harm, including iron overload and transfusion reactions, in addition to substantial burden and costs.

\section{Chronic Pain}

Chronic pain in the guideline is defined as ongoing pain present on most days over the past 6 months.

Recommendation 6. For adult patients with SCD who have chronic pain from the SCD-related identifiable cause avascular necrosis (AVN) of the bone, the ASH guideline panel suggests the use of serotonin-norepinephrine reuptake inhibitors (SNRIs) or NSAIDs in the context of a comprehensive disease and pain management plan (Conditional recommendation; very low certainty in the evidence about effects). For patients with no identifiable cause beyond SCD, the guideline panel suggests SNRIs, tricyclic antidepressants, or gabapentinoids for pain management (Conditional recommendation; very low certainty in the evidence about effects). Given the lack of direct evidence, indirect evidence was used to formulate these recommendations. For pain associated with $A V N$, data were extrapolated from literature on osteoarthritis, a form of degenerative arthropathy. For pain without an identifiable cause, evidence was taken from studies on fibromyalgia, a condition the panel felt most closely aligned with chronic pain related to SCD.

No recommendations were made for pediatric patients as the indirect evidence base only addressed adult patients.

Recommendation 7. For adult and pediatric patients with SCD and emerging and/or recently developed chronic pain, the ASH guideline panel does not recommend initiating COT unless pain is refractory to multiple other treatment modalities (Conditional recommendation; very low certainty in the evidence about effects). For patients receiving COT who are functioning well and have perceived benefit, the ASH guideline panel suggests shared decision-making for continuation of COT (Conditional recommendation; very low certainty in the evidence about effects).

High-quality data on the benefit of long-term COT in individuals with chronic noncancer pain are lacking. The panel maintains that the decision to initiate or continue COT should be individualized after weighing appropriate risks and benefits.

Recommendation 8. For adult and pediatric patients with chronic pain related to SCD, the panel suggests cognitive and behavioral pain management strategies in the context of a comprehensive disease and pain management plan (Conditional recommendation; very low certainty in the evidence about effects). Cognitive behavioral therapy may decrease overall pain intensity and improve coping skills. ${ }^{8}$ The panel agrees that medications alone may not be effective in reducing the burden of chronic pain in adult and pediatric patients with SCD.

\section{CRITIQUE}

The guidelines were created by a multidisciplinary panel that included physicians from hematology, pain medicine, psychiatry, and emergency medicine, a doctoral nurse practitioner, and two patient representatives. The Mayo Evidence-Based Practice Research Program supported the guideline-development process. The GRADE (Grading of Recommendations Assessment, Development, and Evaluation) approach was used to assess evidence and make recommendations.

High-quality data in treating acute and chronic pain in both adult and pediatric patients with SCD are limited. As such, the majority of recommendations in these guidelines are conditional. The panel included studies that were indirectly related to SCD based on consensus (eg, inferred data from disease processes thought to be similar to SCD). One panelist disclosed receiving direct payments from a company that could be affected by these guidelines; however, it was deemed that the conflict was unlikely to have influenced any recommendations.

\section{AREAS IN NEED OF FUTURE STUDY}

The panel acknowledges that further investigation is needed for both nonpharmacologic and pharmacologic modalities in treating acute and chronic pain related to SCD. Examples include evaluating the comparative-effectiveness of COT vs nonopioid pharmacotherapy, the benefits and harms of continuous opioid infusions in acute pain crises, and the impact of chronic transfusions on acute and chronic pain.

Disclosure statement: The authors have nothing to disclose.

\section{References}

1. Data \& statistics on sickle cell disease. Centers for Disease Control and Prevention. Accessed August 23, 2020. https://www.cdc.gov/ncbddd/sicklecell /data.html

2. Complications and treatments of sickle cell disease. Centers for Disease Control and Prevention. Accessed August 23, 2020. https://www.cdc.gov /ncbddd/sicklecell/treatments.html

3. Fingar KR, Owens PL, Reid LD, Mistry KB, Barrett ML. Characteristics of Inpatient Hospital Stays Involving Sickle Cell Disease, 2000-2016. Agency for Healthcare Research and Quality. Healthcare Cost and Utilization Project. Statistical Brief 251. September 2019. Accessed August 23, 2020. www.hcupus.ahrq.gov/reports/statbriefs/sb251-Sickle-Cell-Disease-Stays-2016.pdf

4. Tanabe P, Silva S, Bosworth $\mathrm{HB}$, et al. A randomized controlled trial comparing two vaso-occlusive episode (VOE) protocols in sickle cell disease (SCD). Am J Hematol. 2018;93(2):159-168. https://doi.org/10.1002/ajh.24948

5. Perlin $\mathrm{E}$, Finke $\mathrm{H}$, Castro $\mathrm{O}$, et al. Enhancement of pain control with ketorolac tromethamine in patients with sickle cell vaso-occlusive crisis. Am J Hematol. 1994;46(1):43-47. https://doi.org/10.1002/ajh.2830460108

6. Sheehy KA, Lippold C, Rice AL, et al. Subanesthetic ketamine for pain management in hospitalized children, adolescents, and young adults: a single-center cohort study. J Pain Res. 2017;10:787-795. https://doi.org/10.2147 /jpr.s131156

7. New T, Venable C, Fraser L, et al. Management of refractory pain in hospitalized adolescents with sickle cell disease: changing from intravenous opioids to continuous infusion epidural analgesia. J Pediatr Hematol Oncol. 2014;36(6):e398-e402. https://doi.org/10.1097/mph.0000000000000026

8. Schatz J, Schlenz AM, McClellan CB, et al. Changes in coping, pain, and activity after cognitive-behavioral training: a randomized clinical trial for pediatric sickle cell disease using smartphones. Clin J Pain. 2015;31(6):536-547. https://doi.org/10.1097/ajp.0000000000000183 